\title{
Dimensional doubly dual hyperovals and bent functions
}

\author{
Ulrich Dempwolff
}

\begin{abstract}
We show that dimensional doubly dual hyperovals over $\mathbb{F}_{2}$ define bent functions. We also discuss some known and a few new examples of dimensional doubly dual hyperovals and study the associated bent functions.
\end{abstract}

Keywords : dimensional dual hyperoval, bent function

MSC 2000: 51A45, 05B25

\section{Introduction}

A set $\mathbf{D}$ of $n$-dimensional subspaces of a finite dimensional vector space $V$ over a finite field (say $V=V(m, q)$ ) is called a dual hyperoval of rank $n$, we use the symbol DHO as an abbreviation, if $|\mathbf{D}|=\left(q^{n}-1\right) /(q-1)+1, \operatorname{dim} X \cap Y=1$ and $X \cap Y \cap Z=0$ for three different $X, Y, Z \in \mathbf{D}$. The DHO splits over the subspace $W$ if $V=X \oplus W$ for all $X \in \mathbf{D}$. In this paper we are interested in DHOs, which are also DHOs with respect to the dual space: We call $\mathbf{D}$ a doubly dual hyperoval of rank $n$, we abbreviate DDHO, if $m=2 n$ and $\mathbf{D}$ is also a DHO with respect to the dual space, i.e. if $\operatorname{dim} X+Y=2 n-1$ and $X+Y+Z=V$ for three different $X, Y, Z \in \mathbf{D}$.

Remark 1.1. (i) A set of $\left(q^{n}-1\right) /(q-1)+1$ subspaces of rank $n$ such that any two generate a hyperplane and any three the whole space $V$ is called a dimensional hyperoval in [3] and [8]. So our notion of a DDHO is compatible with the notation in the literature.

(ii) Usually DHOs of rank $n$ are called $(n-1)$-dimensional dual hyperovals. However in our context it seems more natural to use the notions of vector spaces than the language of projective geometry. DHOs are only known for 


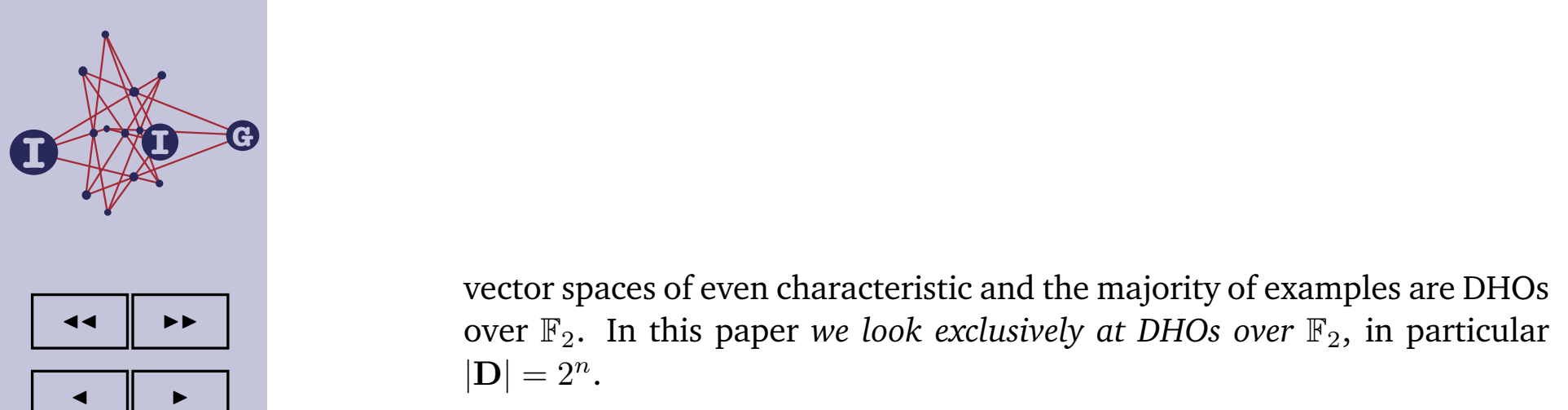

Seemingly unrelated to DHOs is the notion of a bent function. Let $V=$ $V(2 n, 2)$ be an $2 n$-dimensional space over $\mathbb{F}_{2}$. A function $f: V \rightarrow \mathbb{F}_{2}$ is called a bent function, if its support is a difference set in $V$. Equivalently, a function $f$ is bent, if the absolute value of the Fourier transform has the constant value $2^{n}$, i.e. if

$$
|\widehat{f}(v)|=\left|\sum_{x \in V}(-1)^{f(x)+x \cdot v}\right|=2^{n}, \quad v \in V .
$$

There is a vast literature on bent functions. DHOs have been studied intensively too. Yoshiara [23] is a survey article on DHOs. Investigations of DDHOs are contained in Taniguchi [16] and Yoshiara [22].

Starting point of our paper is a connection between DDHOs and bent functions, which is proved in the next section:

Theorem 1.2. Let $\mathbf{D}$ be a dual hyperoval in $V=V(2 n, 2)$ of rank $n$.

(a) Set

$$
B=\left(\bigcup_{S \in \mathbf{D}} S\right)-0
$$

Then the characteristic function of $B$ is bent iff $\mathbf{D}$ is doubly dual.

(b) Assume that $\mathbf{D}$ splits with respect to the subspace $W$. Set

$$
B=W \cup \bigcup_{S \in \mathbf{D}} S .
$$

Then the characteristic function of $B$ is bent iff $\mathbf{D}$ is doubly dual.

This motivates a search for DDHOs. In the third section we define symplectic and orthogonal DHOs (called DHOs of polar type in [22]), discuss their representations and some of their properties. Such DHOs are automatically doubly dual (see Corollary 3.5). In Section 4 we present examples of DDHOs. Some of them are known but others appear to be new. The main emphasis will lie on the construction of symplectic DHOs, which are not orthogonal DHOs. In Section 5 we investigate the bent functions associated to these DDHOs (in the sense of Theorem 1.2). We also will discuss possible isomorphisms between the examples of Section 4.

This article is a predecessor of a forthcoming paper [6] by Kantor and the author. There it is shown that orthogonal DDHOs can be produced in large numbers by projections from orthogonal spreads. This is a variation of Kantor's technique, which uses projections of orthogonal spreads to produce symplectic spreads (see for instance $[12,13,14]$ ). 


\section{Bent functions and doubly dual hyperovals}

Definitions. Set $V=V(m, 2)$. For $f \in \mathcal{F}_{m}=\mathbb{F}_{2}^{V}$ and $x \in V$ define the Fourier coefficient at $v$ by

$$
\widehat{f}(v)=\sum_{x \in V}(-1)^{f(x)+x \cdot v}
$$

go back

full screen

close

quit

and call the function $\widehat{f}: V \rightarrow \mathbf{C}$ the Fourier transform of $f$. We sometimes identify $f$ with its support $\{x \in V \mid f(x)=1\}$ and write $v \in f$ if $f(v)=1$ (i.e. if $v \in f^{-1}(1)$ ). For $v \in V$ define $v^{\perp}: V \rightarrow \mathbb{F}_{2}$ by $v^{\perp}(x)=v \cdot x$ (usual dot product). Finally we set $|f|=\left|f^{-1}(1)\right|$. Let $m=2 n$. The function $f \in \mathcal{F}_{2 n}$ is a bent function if $\widehat{f}(x) \in\left\{ \pm 2^{n}\right\}$ for all $x \in V$. The next two results with their proofs come from [1].

Lemma 2.1. For $f \in \mathcal{F}_{m}$ and $v \in F$ :

(a) $\sum_{x \in V}(-1)^{f(x)}=2^{m}-2|f|$

(b) $\widehat{f}(v)=2^{m}-2\left|f+v^{\perp}\right|$

Proof. We observe

$$
\sum_{x \in V}(-1)^{f(x)}=\sum_{x \in V-f} 1-\sum_{x \in f} 1=2^{m}-2|f|,
$$

which implies (a). From (a) we get $\widehat{f}(v)=\sum_{x}(-1)^{f(x)+v \cdot x}=2^{m}-2\left|f+v^{\perp}\right|$, which is assertion (b).

Proposition 2.2. Let $f \in \mathcal{F}_{2 n}$. Equivalent are:

(a) $f$ is bent.

(b) $\left|f+v^{\perp}\right|=2^{2 n-1} \pm 2^{n-1}$ for all $v \in V$.

(c) There exists $\epsilon \in\{ \pm 1\}$ such that $|f|=2^{2 n-1}+\epsilon 2^{n-1}$ and $\left|f \cap v^{\perp}\right|=2^{2 n-2}+$ $\epsilon 2^{n-1}$ or $=2^{2 n-2}$ for all $0 \neq v \in V$.

Proof. (a) $\Rightarrow$ (b). We have, by Lemma 2.1, $\pm 2^{n}=\widehat{f}(v)=2^{2 n}-2\left|f+v^{\perp}\right|$.

(b) $\Rightarrow$ (c). From (b) we get $|f|=\left|f+0^{\perp}\right|=2^{2 n-1}+\epsilon 2^{n-1}$ with some $\epsilon= \pm 1$. Moreover for $v \neq 0$ we use

$$
\left|f \cap v^{\perp}\right|=\frac{1}{2}\left(|f|+\left|v^{\perp}\right|-\left|f+v^{\perp}\right|\right) .
$$

This implies assertion (c). 
(c) $\Rightarrow(\mathrm{a})$. Now we use

$$
\widehat{f}(v)=2^{2 n}-2\left|f+v^{\perp}\right|=2^{2 n}-2\left(|f|+\left|v^{\perp}\right|-2\left|f \cap v^{\perp}\right|\right) .
$$

Using (c) we get assertion (a).

We use Proposition 2.2 to verify Theorem 1.2:

Proof of Theorem 1.2. First we note, that $|\mathbf{D}|=2^{n}$ by the definition of a DHO, and that every nontrivial vector of $V$ lies either in 2 or none elements from $\mathbf{D}$.

full screen

close

quit

(a) An application of the sieve formula shows $|B|=2^{2 n-1}-2^{n-1}$. Let $v \in V-0$. Let $S_{1}, \ldots, S_{k}$ be the spaces of $\mathbf{D}$ which are in $v^{\perp}$; note that $k$ can be 0 . Set $B_{0}=\left(S_{1} \cup \cdots \cup S_{k}\right)-0$. By the sieve formula

$$
\left|B_{0}\right|=k\left(2^{n}-1\right)-\left(\begin{array}{l}
k \\
2
\end{array}\right)=\frac{k}{2}\left(2^{n+1}-k-1\right) .
$$

Set $\mathbf{D}^{\prime}=\mathbf{D}-\left\{S_{1}, \ldots, S_{k}\right\}$. Then $\left|S^{\prime} \cap B_{0}\right|=k$ for $S^{\prime} \in \mathbf{D}^{\prime}$. Set $B_{1}=(B \cap$ $\left.v^{\perp}\right)-B_{0}$. Since $\left|S^{\prime} \cap v^{\perp}\right|=2^{n-1}-1$ we see $\left|B_{1} \cap S^{\prime}\right|=2^{n-1}-k-1$. Consider the incidence structure $\left(B_{1}, \mathbf{D}^{\prime}\right)$. We deduce $2\left|B_{1}\right|=\left(2^{n}-k\right)\left(2^{n-1}-k-1\right)$. This implies

$$
\left|B \cap v^{\perp}\right|=\left|B_{0}\right|+\left|B_{1}\right|=2^{2 n-2}+2^{n-2}(k-2) .
$$

If $\mathbf{D}$ is doubly dual then always $k=0$ or $=2$, i.e. $\left|B \cap v^{\perp}\right|=2^{2 n-2}$ or $=2^{2 n-1}-2^{n-1}$. Hence $f_{B}$ is bent by Proposition 2.2. If $\mathbf{D}$ is not doubly dual, then we can choose $v$ such that $0 \neq k \neq 2$. Then $\left|B \cap v^{\perp}\right| \neq 2^{2 n-2}$ and $\neq 2^{2 n-2}-2^{n-1}$. So $f_{B}$ is not bent in this case.

(b) Now $B$ is partitioned into $W$ and $\left(\bigcup_{S \in \mathbf{D}} S\right)-0$. We deduce from (a) that $|B|=2^{2 n-1}+2^{n-1}$.

Let $v \in V-0$. Assume first $W \subseteq v^{\perp}$. Then $S \nsubseteq v^{\perp}$ for all $S \in \mathbf{D}$. As $B \cap v^{\perp}$ is partitioned into $W$ and $\left(\left(\bigcup_{S \in \mathbf{D}} S\right)-0\right) \cap v^{\perp}$ we deduce from (a) $\left|B \cap v^{\perp}\right|=2^{n}+2^{2 n-2}-2^{n-1}=2^{2 n-2}+2^{n-1}$.

Assume next $W \nsubseteq v^{\perp}$. Then $\left|W \cap v^{\perp}\right|=2^{n-1}$. If $v^{\perp}$ contains precisely $k$ spaces from $\mathbf{D}$, we deduce from (a)

$$
\left|B \cap v^{\perp}\right|=2^{2 n-2}+2^{n-2}(k-2)+2^{n-1} .
$$

If $\mathbf{D}$ is doubly dual, we have $k=0$ or 2 and $\left|B \cap v^{\perp}\right|=2^{2 n-2}$ or $=2^{2 n-2}+$ $2^{n-1}$. Again $f_{B}$ is bent by Proposition 2.2. If however $\mathbf{D}$ is not doubly dual, we can choose $v$ such that $0 \neq k \neq 2$. Thus $\left|B \cap v^{\perp}\right| \neq 2^{2 n-2}$ nor $\neq 2^{2 n-2}+2^{n-1}$ and $f_{B}$ is not bent in this case. 





Proposition 4.2 (S. Yoshiara). Let $(t, n)=(2 r, n)=(2 r+t, n)=1$. Then the DHO $\mathbf{D}_{r, t}$ is symplectic iff $t=-r$.

Proof. By Lemma 3.1 the DDHO $\mathbf{D}_{r, t}$ can only be symplectic if there exists a $A \in \mathrm{GL}_{\mathbb{F}_{2}}(F)$ such that

$$
A B(y)^{\star}=B(y) A^{\star} \quad \text { for all } \quad y \in F .
$$

go back

full screen

close

quit

Let $A=\sum_{i=0}^{n-1} T_{i}\left(a_{i}\right)$. Then $A^{\star}=\sum_{i=0}^{n-1} T_{i}\left(a_{-i}^{2^{-i}}\right)$ and $B(y)^{\star}=T_{r}\left(y^{2^{t+r}}\right)+$ $T_{-r}\left(y^{2^{-r}}\right)$. Evaluating the condition $B(y) A^{\star}=A B(y)^{\star}$ leads to the equations

$$
a_{i-r}^{2^{r}} y^{2^{r+t}}+a_{i+r}^{2^{-r}} y^{2^{-r}}=a_{r-i}^{2^{r-i}} y^{2^{i-r}}+a_{-i-r}^{2^{-i-r}} y^{2^{i+r+t}} \quad \text { for all } \quad y \in F
$$

and $0 \leq i<n$. The coefficients in such an equation can only be nontrivial if exponents of $y$ on the LHS occur also on the RHS, i.e. one has $i=0, \pm(r+t)$. Inspecting the equation for $i=2 r+t$ shows $a_{3 r+t}=a_{-3 r-t}=0$. Then these three equations show that the only nontrivial coefficients can be $a_{ \pm r}$ or $a_{ \pm(r+t)}$. The equation for $i=2 r$ involves $a_{ \pm r}$ and it follows $a_{r}=a_{-r}=0$ as the respective exponents of $y$ for these coefficient occur only once in this equation. So the only possible nontrivial coefficients are $a_{r+t}$ and $a_{-r-t}$. The remaining equations which involve these coefficients occur for $i= \pm t$. An inspection of these two equations shows, that $a_{r+t}$ or $a_{-r-t}$ can only be nontrivial if $r=-t$ and $a_{0}^{2^{t}}=a_{0}$. But we observed already that for $r=-t$ the DHO $\mathbf{D}_{r,-r}$ is symplectic. The proof is complete.

The next four examples describe bilinear DHOs, which are symplectic but not orthogonal (see Proposition 4.16). The symmetric Knuth image of the first example has been studied already by Taniguchi and Yoshiara [19].

Example 4.3. For $y \in F$ we define a $\mathbb{F}_{2}$-linear mapping $B(y)$ on $F$ by

$$
x B(y)=x\left(y+y^{2^{n-1}}\right)+x^{4} y+(x y)^{2^{n-2}} .
$$

Set

$$
\mathbf{D}=\left\{S_{y} \mid y \in F\right\}, \quad S_{y}=\{(x, x B(y)) \mid x \in F\} .
$$

That $\mathbf{D}$ is a symplectic DDHO follows from:

Lemma 4.4. The set $\mathbf{D}$ is a symplectic, bilinear $D H O$ on $V$.

Proof. Clearly, the operators $B(y)$ are all self-adjoint with respect to the trace form. So we have only to show that $\mathbf{D}$ is a DHO, i.e. we have to show that each $B(y), y \neq 0$ has rank $n-1$ and that $\operatorname{ker} B(y) \neq \operatorname{ker} B(z)$ for $0 \neq y \neq z \neq 0$. Now 

This shows that that the symmetric DHO is a DHO described by Taniguchi and Yoshiara in [19, Sec. 3]. The verification that the mapping $B^{o *}$ defines a DHO in [19] is simpler than our verification of the DHO property. But the proof of Taniguchi and Yoshiara does not show that $B^{o *}$ defines a doubly dual DHO. So our result needs a separate verification.

Example 4.6. For $y \in F$ we define a $\mathbb{F}_{2}$-linear mapping $B(y)$ on $F$ by

$$
x B(y)=x(y+\operatorname{Tr}(y))+x^{2} y+(x y)^{2^{n-1}} .
$$

Set

$$
\mathbf{D}=\left\{S_{y} \mid y \in F\right\}, \quad S_{y}=\{(x, x B(y)) \mid x \in F\} .
$$

Then $\mathbf{D}$ is a symplectic, bilinear DDHO. This follows from follows from:

Lemma 4.7. The set $\mathbf{D}$ is a symplectic, bilinear $D H O$ on $V$.

Proof. Clearly, all operators $B(y)$ are self-adjoint with respect to the trace form. We have to show that each $B(y), y \neq 0$ has rank $n-1$ and that ker $B(y) \neq$ $\operatorname{ker} B(z)$ for $0 \neq y \neq z \neq 0$. Now $x \in \operatorname{ker} B(y)$ iff $x(y+\operatorname{Tr}(y))+x^{2} y+(x y)^{2^{n-1}}=$ 0 or after squaring we see that $(x, y)$ is a root of

$$
f(X, Y)=X Y+(X Y)^{2}+X^{2} \operatorname{Tr}(Y)+X^{4} Y^{2} .
$$

Clearly, for $y=1$ we get $x=1$ as the unique root of $f(X, 1)$. So we assume from now on $y \in F-\{0,1\}$.

For the case $\operatorname{Tr}(y)=0$ we substitute the variable $Y$ by $Y^{2}+Y$ in $f(X, Y)$ and obtain the polynomial $f_{0}(X, Y)=f\left(X, Y^{2}+Y\right)$, which factorizes as $f_{0}(X, Y)=$ $X Y(Y+1) g(X, Y)$ with

$$
g(X, Y)=X^{3} Y^{2}+X^{3} Y+X Y^{2}+X Y+1=Y^{2}\left(X^{3}+X\right)+Y\left(X^{3}+X\right)+1 .
$$

Note that this polynomial has no roots of the form $(1, y)$. Multiply with $X^{-3}$ and substitute $X$ by $Z=X^{-1}$. Then

$$
g(Z, Y)=Z^{3}+\left(Y^{2}+Y\right) Z^{2}+Y^{2}+Y .
$$

So for a solution $(z, y) \in(F-\{0,1\})^{2}$ we have $y^{2}+y=\frac{z^{3}}{z^{2}+1}$. Substitute $u=\frac{1}{1+z}$. Then $y^{2}+y=u^{2}\left(\frac{u+1}{u}\right)^{3}=u^{2}+u+1+u^{-1}$. Hence $\operatorname{Tr}\left(u^{-1}\right)=1$ or $\operatorname{Tr}\left(x^{-1}\right)=\operatorname{Tr}(z)=0$ where $x=z^{-1}$. We set $F_{0}=\left\{x \in F^{\star} \mid \operatorname{Tr}(x)=0\right\}$. We have already seen that the mapping $\phi$

$$
F_{0} \ni z \mapsto \phi(z)=\frac{z^{3}}{z^{2}+1}
$$





We first prove claim (1). The polynomial $h(X, Y)=\left(X^{5}+X^{3}+X+1\right)\left(Y^{4}+\right.$ $\left.Y^{2}+1\right)+\left(Y^{5}+Y^{3}+Y+1\right)\left(X^{4}+X^{2}+1\right)$ factorizes over $E=\mathbb{F}_{2^{2 n}}$ as $h(X, Y)=$ $(X+Y) h_{1}(X, Y) h_{-1}(X, Y)$ with

$$
h_{i}(X, Y)=X^{2} Y^{2}+\nu^{i} X^{2}+X+\nu^{i} Y^{2}+Y+\nu^{i}
$$

for $i= \pm 1$. If $\phi\left(z_{1}\right)=\phi\left(z_{2}\right)$, then $\left(z_{1}, z_{2}\right)$ is a root of the polynomial $h(X, Y)$. Assume $z_{1} \neq z_{2}$. Then $\left(z_{1}, z_{2}\right)$ is a root of one of the $h_{i}(X, Y)$ 's and so of both of them. We get

$$
0=h_{1}\left(z_{1}, z_{2}\right)+h_{-1}\left(z_{1}, z_{2}\right)=z_{1}^{2}+z_{2}^{2}+1, \quad \text { or } \quad z_{2}=z_{1}+1 .
$$

But $h_{1}\left(z_{1}, z_{1}+1\right)=z_{1}^{4}+z_{1}^{2}+1=0$ which is impossible. So (1) holds.

We now prove claim (2). If (2) holds, then $\left(z_{1}, z_{2}\right)$ is a root of the polynomial $\ell(X, Y)=h(X, Y)+\left(X^{4}+X^{2}+1\right)\left(Y^{4}+Y^{2}+1\right)$, which factorizes over $E$ as $\ell(X, Y)=(X+Y+1) \ell_{1}(X, Y) \ell_{-1}(X, Y)$ with

$$
\ell_{i}(X, Y)=X^{2} Y^{2}+\nu^{i} X^{2}+X+\nu^{i} Y^{2}+Y+1
$$

for $i= \pm 1$. If $\left(z_{1}, z_{2}\right)$ is a root of one $\ell_{i}(X, Y)$, then, as usual, it is a root of both factors. This implies

$$
0=\ell_{1}\left(z_{1}, z_{2}\right)+\ell_{-1}\left(z_{1}, z_{2}\right)=z_{1}^{2}+z_{2}^{2}, \quad \text { or } \quad z_{2}=z_{1} .
$$

But $\ell_{1}\left(z_{1}, z_{1}\right)=z_{1}^{4}+1=0$, which is impossible. So (2) holds.

From case 1 and 2 we conclude that for $y \in F_{0}$ there is a unique $x=x_{y} \neq 0$ such that $(x, y)$ is a solution of equation $(*)$. Moreover $x_{y} \in F_{0}$ and $\operatorname{Tr}\left(x^{-1}\right)=0$, if $\operatorname{Tr}(y)=0$ and $\operatorname{Tr}\left(x^{-1}\right)=1$, if $\operatorname{Tr}(y)=1$. Then using (1) and (2) we conclude that the mapping $y \mapsto x_{y}$ is a bijection of $F^{\star}$. The proof is complete.

Remark 4.11. Using the Knuth operations we obtain a symmetric, bilinear DHO defined by $B^{o *}$. It has the form

$$
x B^{o *}(y)=x y+x^{2} y+x y^{2}+\operatorname{Tr}\left(x^{4} y+x^{2} y+x y+x y^{2}+x y^{4}\right) .
$$

Example 4.12. For $y \in F$ we define a $\mathbb{F}_{2}$-linear, self-adjoint mapping $B(y)$ on $F$ by

$$
x B(y)=x \operatorname{Tr}(y)+\operatorname{Tr}(x) y^{2^{n-2}}+\operatorname{Tr}\left(x^{4} y\right)+x y^{2^{n-1}}+x^{2} y^{2^{n-2}}+x^{2^{n-1}} y^{2^{n-3}} .
$$

Set

$$
\mathbf{D}=\left\{S_{y} \mid y \in F\right\}, \quad S_{y}=\{(x, x B(y)) \mid x \in F\} .
$$

Then $\mathbf{D}$ is a symplectic, bilinear DDHO: Taking the 8-th power of the equation $0=x B(y)$ and replacing (by abusing the notation) $x^{4}$ by $x$ we obtain

$$
0=x^{2} \operatorname{Tr}(y)+\operatorname{Tr}(x) y^{2}+\operatorname{Tr}(x y)+x^{4} y^{2}+x^{2} y^{4}+x y .
$$

The assertion follows from two results of Peter Müller [15]. 


Similarly, for $\operatorname{Tr}(y)=1$ we have to consider the curve $X^{3} Y^{2}+X Y^{4}+X+Y=0$ which is parametrized by

$$
x=\frac{(t+1)^{3}}{t}, \quad y=\frac{1}{t^{2}+t}=\frac{1}{t}+\frac{1}{t+1} .
$$

By Lemma $4.14($ as $\operatorname{Tr}(x)=0)$ there is a $t \in F^{\star}$ with $x=\frac{(t+1)^{3}}{t}=t^{2}+t+1+\frac{1}{t}$. Set

$$
y_{1}=\frac{t^{3}}{t+1}=t^{2}+t+1+\frac{1}{t+1} \quad \text { and } \quad y_{2}=\frac{1}{t^{2}+t}=\frac{1}{t}+\frac{1}{t+1} .
$$

The condition $\operatorname{Tr}(x)=0$ implies $\operatorname{Tr}\left(\frac{1}{t}\right)=1$. Thus

$$
\operatorname{Tr}\left(y_{1}\right)=1+\operatorname{Tr}\left(\frac{1}{t+1}\right) \text { and } \operatorname{Tr}\left(y_{2}\right)=1+\operatorname{Tr}\left(\frac{1}{t+1}\right)=\operatorname{Tr}\left(y_{1}\right) .
$$

So if $\operatorname{Tr}\left(\frac{1}{t+1}\right)=0$, then $\operatorname{Tr}\left(y_{2}\right)=1$ and we set $y=y_{2}$. If $\operatorname{Tr}\left(\frac{1}{t+1}\right)=1$, then $\operatorname{Tr}\left(y_{1}\right)=0$ and we set $y=y_{1}$. At any rate, we get a solution $y$ of $f(x, Y)=0$ in the case of $\operatorname{Tr}(x)=0$.

We need to show the uniqueness of $y$. Suppose there is another $y^{\prime}$ with $f\left(x, y^{\prime}\right)=0$. From the parametrizations, which give in both cases a bijection between the pairs $(x, y)$ and the parameter $t$, it is clear that $y$ and $y^{\prime}$ must correspond to different cases. So $\operatorname{Tr}(y) \neq \operatorname{Tr}\left(y^{\prime}\right)$. By Lemma $4.14 t$ is uniquely determined by $x$. So the parameter $t$ is the same for $y$ and $y^{\prime}$. But then $\operatorname{Tr}(y)=$ $\operatorname{Tr}\left(y^{\prime}\right)$ (as we have seen above), a contradiction. The proof is complete.

Remark 4.15. Since our DHO is bilinear, one can use the Knuth operations and obtains a symmetric, bilinear DHO $B^{o *}$ of the form

$$
x B^{o *}(y)=x^{4} \operatorname{Tr}(y)+\operatorname{Tr}(x) y^{4}+\operatorname{Tr}(x y)+x^{2} y^{2}+x^{8} y^{4}+x^{4} y^{8} .
$$

Proposition 4.16. The DHOs of Examples 4.3, 4.6, 4.9, and 4.12 are symplectic but not orthogonal.

Proof. Apply Lemma 3.10, Lemma 3.11 and Remark 3.12.

There is an alternative way to verify this fact: Use the associated symmetric DHOs obtained from the Knuth operations. If one of our DHOs would be orthogonal, its associated symmetric DHO would even be alternating. Assume that the additive map $B^{\prime}: F \rightarrow \operatorname{End}(F)$ defines the additively closed DHO-set of this alternating DHO. Then define a mapping $\kappa: F \rightarrow F$ by setting $\kappa(0)=0$ and defining $\kappa(a)$ as the nontrivial element in $\operatorname{ker} B^{\prime}(a)$ for $a \neq 0$. Then by Taniguchis [17, Prop. 3] $\kappa$ would be linear. So one needs to rule out the linearity of $\kappa$. However to verify this non-linearity seems to be somewhat unpleasant.

We close this section with a simple series of orthogonal, non-bilinear DHOs. 





Firstly,

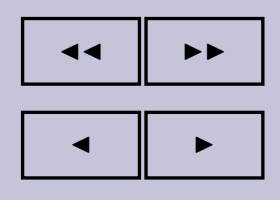

page 27 / 30

go back

full screen

close

quit

$$
\begin{aligned}
\operatorname{Tr}(x \cdot x B(y))= & \operatorname{Tr}\left(x^{2} \operatorname{Tr}(y)+x y^{2^{n-2}} \operatorname{Tr}(x)+x \operatorname{Tr}\left(x^{4} y\right)\right. \\
& \left.\quad+x^{2} y^{2^{n-1}}+x^{3} y^{2^{n-2}}+x^{2^{n-1}+1} y^{2^{n-3}}\right) \\
= & \operatorname{Tr}(x) \operatorname{Tr}(y)+\operatorname{Tr}\left(x^{2} y^{2^{n-1}}\right) \\
= & \operatorname{Tr}\left(x\left(\operatorname{Tr}(y)+y^{2^{n-2}}\right)\right)
\end{aligned}
$$

and secondly

$$
\begin{aligned}
\operatorname{Tr}(x B(y))= & \operatorname{Tr}\left(x \operatorname{Tr}(y)+y^{2^{n-2}} \operatorname{Tr}(x)+\operatorname{Tr}\left(x^{4} y\right)\right. \\
& \left.\quad+x y^{2^{n-1}}+x^{2} y^{2^{n-2}}+x^{2^{n-1}} y^{2^{n-3}}\right) \\
= & \operatorname{Tr}\left(x\left(y^{2^{n-1}}+y^{2^{n-3}}\right)\right) .
\end{aligned}
$$

Clearly, the mapping $y \mapsto \operatorname{Tr}(y)+y^{2^{n-2}}+y^{2^{n-1}}+y^{2^{n-3}}$ has non-zero values (consider the degree of this polynomial). So for such a $y$ we can choose $x$ such that

$$
1=\operatorname{Tr}\left(x\left(\operatorname{Tr}(y)+y^{2^{n-2}}+y^{2^{n-1}}+y^{2^{n-3}}\right)\right)=Q_{1}(x, x B(y)) .
$$

But this contradicts the assumption, the proof is complete.

Isomorphisms. We now show that the examples of Section 4 produce essentially nonisomorphic DHOs.

First of all, the DHOs $\mathbf{D}_{r, t}, r \neq-t$, form Example 4.1 cannot be isomorphic to DHOs $\mathbf{D}_{r,-r}$ or to DHOs from Examples 4.3, 4.6, 4.9, 4.12, or 4.17 by Lemma 4.2. The orthogonal DHOs from Example 4.17 cannot be isommorphic to the examples from 4.1, 4.3, 4.6, 4.9, or 4.12 by Lemma 4.19. Also the isomorphism problem for two DHOs from Example 4.1 has been solved in [21] and [18].

Since the DHOs from Examples 4.3, 4.6, 4.9, 4.12 are symplectic but not orthogonal (Proposition 4.16) we only have to exclude isomorphisms between these examples. A possible isomorphism between DDHOs maps the support of one small bent function to the support of the small bent function of the other DDHO. So by Proposition 5.5 and Lemma 5.6 the DHOs from Examples 4.3, 4.6, and 4.9 cannot be isomorphic to DHOs from Example 4.12.

Finally, to a DHO D on $V$ one can associate a semi-biplane $(V, \mathbf{L}), \mathbf{L}=\{S+v \mid$ $S \in \mathbf{D}, v \in V\}$. For the Examples 4.3, 4.6, and 4.9 and dimensions $n=5$ and 7 we calculated with the help of a computer the 2-rank of the incidence matrix:

\begin{tabular}{|c|c|c|c|}
\hline$n$ & Example 4.3 & Example 4.6 & Example 4.9 \\
\hline 5 & 326 & 327 & 326 \\
7 & 3766 & 3818 & 3828 \\
\hline
\end{tabular}


